\title{
Machine Learning Cases in Clinical and Biomedical Domains
}

Mohd Zulfaezal Che Azemin ${ }^{1}$,Tijani Ahmed Ashimi ${ }^{2}$, Md Muziman Syah ${ }^{2}$

${ }^{1}$ Department of Optometry and Visual Science, Kulliyyah of Allied Health Sciences, International Islamic University Malaysia, Malaysia ${ }^{2}$ Kulliyyah of Islamic Revealed Knowledge and Human Sciences, International Islamic University Malaysia, Malaysia

\section{ABSTRACT}

The aim of this paper is twofold: Firstly, to provide introductory knowledge to the reader who has little or no knowledge of machine learning with examples of applications in clinical and biomedical domains, and secondly, to compare and contrast the concept of Artificial Neural Network (ANN) and the Qur'anic concept of intellect (aql) in the Qur'an. Learning algorithm can generally be categorised into supervised and unsupervised learning. To better understand the machine learning concept, hypothetical data of glaucoma cases are presented. ANN is then selected as an example of supervised learning and the underlying principles in ANN are presented with general audience in mind with an attempt to relate the mechanism employed in the algorithm with Qur'anic verses containing the verbs derived from aql. The applications of machine learning in clinical and biomedical domains are briefly demonstrated based on the author's own research and most recent examples available from University of California, Irvine Machine Learning Repository. Selected verses which indicate motivation to use the intellect in positive manners and rebuke to those who do not activate the intellect are presented. The evidence found from the verses suggests that ANN shares similar learning process to achieve belief (iman) by analysing the similitudes (amsal) introduced to the algorithm.

KEYWORDS: Machine Learning; Artificial Neural Network; Aql; Intellect; Dataset

\section{INTRODUCTION}

When making clinical judgment, a person employs various sensory stimuli which may include vision, auditory, somatic and olfactory senses to assist with diagnosis. These complex sensory inputs are then fed into higher order processing areas in the brain. Through years of training to understand a specific pattern under supervision of experts, specialised clinical or medical skills may be transferred. Machine learning based on Artificial Neural Network (ANN) algorithm is a practical example of inspiration that has been drawn to adopt the learning process derived from the complexity of the human brain and nervous system.

Algorithm with an ability to learn from experience; this may be a dumbed-down definition of machine learning which is commonly associated with automation. Without realizing, learning algorithms are often integrated in our daily usage of technologies. When we use Google or Bing, the search engines will try to sort web pages with an ultimate goal of matching it with desired information. Behind the simplified text box query, machine learning is actually at work here. Spam email is a common problem for many people and

Mohd Zulfaezal Che Azemin Department of Optometry and Visual Science, Kulliyyah of Allied Health Sciences, International Islamic University Malaysia, Kuantan Campus.

E-mail:zulfaezal@iium.edu.my thanks to learning algorithm e-mail application can automatically classify the spam e-mails and redirect them to a special folder. Self-driving car is no longer a thing of the future as demonstrated by Google car which has clocked up over a million kilometres with no reported major accident. Tesla Motors has brought the concept to another level by making it available to the masses. This is made possible with fusion of sensors' data mapped by machine learning techniques.

In the biomedical domain, cancer detection accuracy has shown significant improvements using inputs from gene expression profiling, histological and clinical data fed into machine learning framework. ${ }^{1}$ More than 7,000 scientific articles have been published on the use of learning algorithms utilizing data from various sources for detection of cancer types and tumours. ${ }^{1}$ In one research, ${ }^{2}$ it was shown that using only 16 DNA methylation markers, a machine learning model can be used to classify lung cancer with an accuracy of $86 \%$ into its major types; non-small cell lung cancer, small cell lung cancer and carcinoid. This kind of research has grown exponentially owing to significant increase in computer processing power with affordable cost. If in yesteryears, this type of study only confined to well-funded institutions, now it is accessible to almost all researchers with decent computers and the right machine learning tools.

Dr. Andrew Ng, an Associate Professor at Stanford University describes machine learning using a game of checkers; a computer program is said to learn 
from playing 10's of 1000's of checker games against itself, and the performance measure will be the probability that it wins the next game against new opponents. ${ }^{3}$ Historic events had taken place between 1996 to 1997, six-game chess matches between world chess champion Garry Kasparov and IBM supercomputer known as Deep Blue. ${ }^{4}$ While Deep Blue won the second game, the computer relied on brute force algorithm which evaluates millions of possible moves. Google's AlphaGo on the other hand, won a Chinese traditional game called Go against human by relying on neural network, a type of machine learning algorithm. The major difference between these two computers is the latter employs reinforcement learning to maximize its performance while the former does not. ${ }^{5}$

\section{Examples of Machine Learning Cases in Clinical and Biomedical Domains}

This section describes some examples of the application of machine learning in medical and biomedical cases. The first two cases are instances taken from the author's own research and the rest of the cases are the most recent examples available from UCI Machine Learning Repository.

Example 1: Stroke Prediction from Retina Images ${ }^{7}$ In this paper, it was shown that using retina images captured years prior to stroke events, the learning algorithm, specifically, logistic regression model was able to predict with the sensitivity (i.e. ability to correctly identify stroke cases) and specificity (i.e. ability to correctly identify control cases) of $72.52 \%$ and $69.67 \%$, respectively. The research used the data collected from the Blue Mountains Eye Study (BMES), a population-based cohort study of eye diseases and other health outcomes in a suburban Australian population with age range of 50-89 years. The retina images were enhanced at different wavelet scales, and processed with textural analysis known as Fourier Fractal Dimension (FFD). For each retina image, it would have up to four FFD measurements, computed from the enhanced images. The FFD values were used as inputs and the stroke/non-stroke cases as labels.

\section{Example 2: Automatic Redness Measurement from Fibrovascular Tissue ${ }^{8-9}$}

The research work aimed to automatically grade redness level of wing-shaped growth in the eye. The supervised learning employed in this research was Generalized Regression Neural Network. The research used different colour spaces (e.g., YPbPr, $\mathrm{CIE} L A B$ and $\mathrm{CIE} X Y Z$ ) as opposed to the traditional Red-Green-Blue as inputs to the supervised model. The images from multiple colour spaces were then analysed using a statistical measure, entropy, a measure of randomness to characterise the texture of the image. The results from this study indicated the supervised model was able to closely mimic human's grading with minimal error. A recent clinical publication has demonstrated the application of this automated grading in predicting contrast sensitivity function (CSF) and visual acuity (VA) following pterygium surgery. It was shown that the redness grading was able to predict $23 \%$ and $25 \%$ of the variance in the CSF and the VA, respectively. ${ }^{9}$

\section{Example 3: Mice Protein Expression Dataset ${ }^{10}$}

This dataset comprises of 8 labelled groups of mice; 4 of controls and 4 of Down syndrome labels. The objective of this dataset is to identify a subset of 77 proteins that are able to dichotomously categorise the mice as controls or Down syndrome cases. While only using 72 mice, each mouse was subjected to 15 measurements, yielding a total of $1080(72 \times 15)$ measurements per protein. Using Self-Organising Map (SOM), a type of unsupervised learning approach, previous research work was able to identify critical groups of proteins that contributed to successful learning. Further research is warranted in identifying subset protein profiles which may assist in the development of more potent drug.

\section{Example 4: HIV-1 Protease Cleavage Dataset ${ }^{11}$}

Protease inhibitor $(\mathrm{PI})$ is a common strategy used in developing antiviral drugs for human immunodeficiency virus (HIV). More effective PI requires specificity of the HIV type 1 (HIV-1) protease cleavage. The dataset contains subsets of 746 and 1625 peptides that had been experimentally validated for HIV-1 protease cleavage. Based on supervised learning models including support vector machines and neural networks, the research found two physicochemical attributes for the amino acids that are critical to accurately predict the protease cleavage.

\section{Example 5: Diabetic Retinopathy Debrecen Dataset $^{12}$}

Diabetic retinopathy is characterised by specific signs visible in retina images. This dataset provides features extracted from retina images for diabetic retinopathy classification. The features include the characteristics of lesions (e.g. exudate), anatomical components (e.g. optic disc, macula) and also image processing features (e.g. pixel intensity, geometry, textural analysis). The research showed that it was able to automatically classify images with diabetic retinopathy with $90 \%$ sensitivity and $91 \%$ specificity.

\section{Parkinson Speech Dataset ${ }^{13}$}

This Parkinson database comprises of 2 sets of data; one for training and another for testing. The first set contains different types of voice recordings include sustained vowels, numbers, words and short sentences. The features were extracted from linear and time-frequency analyses. The labels for each participant were obtained from Unified Parkinson's disease Rating Scale score which was determined by certified physician. The testing dataset only includes recordings of sustained vowels ' $a$ ' and ' 0 ' three times. This independent dataset is important to validate the supervised model formulated using the training data. 


\section{METHOD}

This section is divided into two parts. The first part briefly explains the basic concept of machine learning using a hypothetical data. The second part compares and contrasts the concept of Artificial Neural Network (ANN) and the Qur'anic concept of intellect (aql).

\section{Types of Learning Algorithm}

The following introductory content of machine learning has been adopted from lectures by $\mathrm{Dr}$. Andrew $\mathrm{Ng}^{3}{ }^{3}$ Learning algorithm can generally be categorised into supervised and unsupervised learning. The first category requires the data to have a label and the second category does not. One example is in the case of cancer diagnosis, supervised learning needs historical data with confirmed cancer cases possibly through pathologic diagnosis from which the computer programme learns to predict cancer or non-cancer instances. In unsupervised learning, the algorithm will try to find underlying pattern from the data and grouped the data into different classes without the labelled cancer cases.

\section{Supervised Learning}

To better understand the concept of supervised learning, hypothetical data of glaucoma cases will be used. Glaucoma is one of the leading causes of blindness with multiple risk factors. One of the parameters that can be used to diagnose glaucoma is intraocular pressure (IOP), the pressure within the eyeball. Figure 1 illustrates a historical dataset collected from medical records. In this example, the horizontal axis represents the IOP and the vertical axis is plotted as one or zero, glaucoma or non-glaucoma cases. The figure illustrates four instances of glaucoma cases indicated by the vertical axis value of one and six instances of healthy individuals with vertical axis value of zero. In machine learning, this is an example of classification problem, particularly a two-label classification problem with the task of predicting dichotomous outcomes; glaucoma or non-glaucoma cases. In other instances, we can have more than one labels to predict, as an extension to the example in Figure 1 we may have different types of glaucoma that we want to predict. The labels may be updated to discrete values of zero, one, or two with one and two assigned for different types of

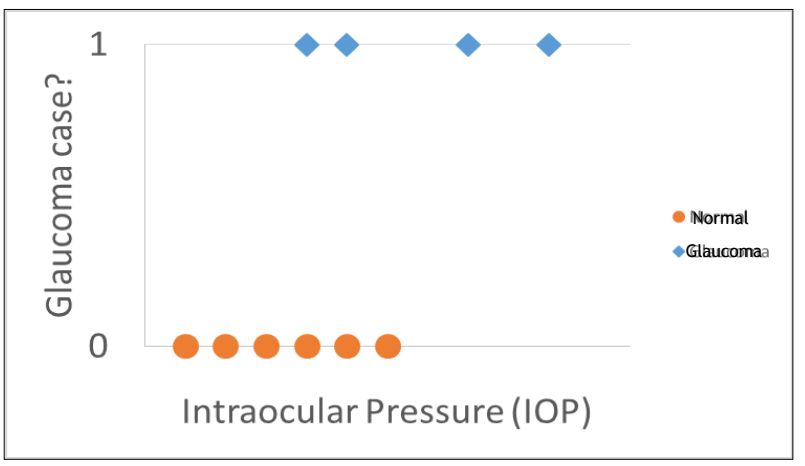

Figure 1: Hypothetical dataset of glaucoma cases plotted against intraocular pressure. glaucoma disease.

Based on the IOP inputs as shown in Figure 2, a dashed line can be drawn to discriminate between the two cases with a modest degree of accuracy. This is an example of classification problem using historical data based on a single feature (i.e. IOP) with the aim of predicting whether the eye is normal or glaucomatous. It is then possible to use machine learning to predict the probability of having glaucoma for a new patient given the pressure inside the eyeball.

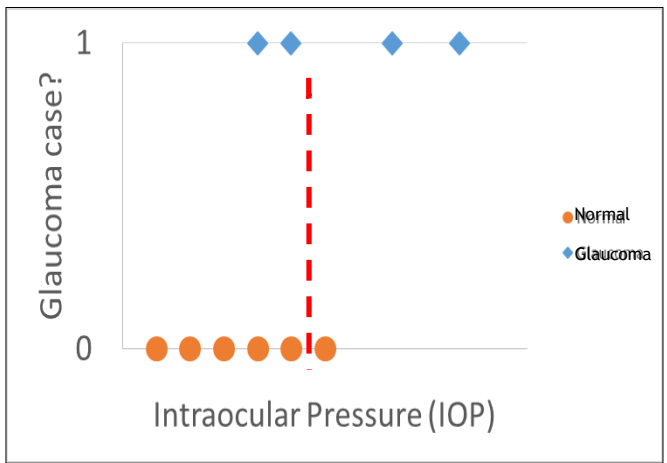

Figure 2: Two-label classification problem with a single feature.

In most cases, machine learning attempts to use information using more than one feature. In Figure 3 , age is now added as a new feature. A learning algorithm then would estimate position of the dashed line that can best separate between glaucomatous and non-glaucomatous cases. When a new patient comes in marked as ' $X$ ' in the figure, it is possible to evaluate that the patient is more likely to be normal than glaucomatous.

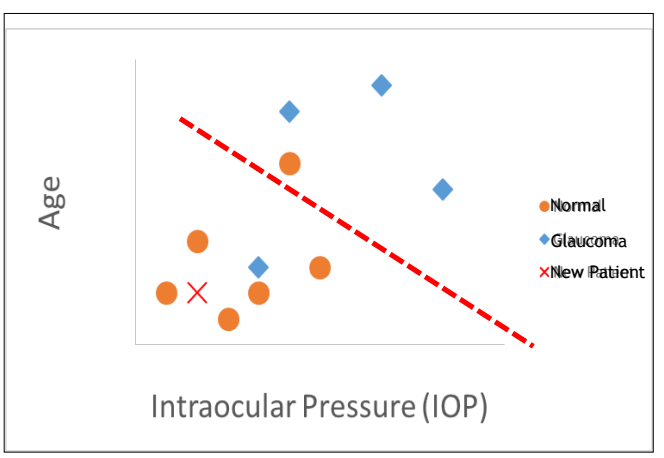

Figure 3: Classification problem with two features.

The accuracy of the prediction may further be improved by incorporating more features. In this example, more information can be added from other diagnoses which include optic nerve and retinal nerve fibre imaging, and results from visual field test. Machine learning algorithms commonly able to process more features which in certain cases can reach thousands of features. ${ }^{14}$ DOROTHEA, a drug discovery dataset for instance, contains 100,000 features with an ultimate goal of separating active from inactive compounds. Another example is ARCENE, a study aimed to classify cancer and healthy individuals using 10,000 features extracted from mass-spectrometric data. 


\section{Unsupervised Learning}

Another type of machine learning algorithm seeks to find similar pattern from a set of data without given labels. This type of learning is called unsupervised learning. The most popular is clustering technique which attempts to find underlying patterns from a given dataset and group the data into statistically similar clusters. In the previous hypothetical example, an unsupervised algorithm may group the data as illustrated in Figure 4. Assuming there is a new patient with a certain age and IOP, marked with ' $X$ ' in the figure; we can deduce that he or she belongs to Cluster 2, which may be categorised as group with higher risk of having glaucoma.

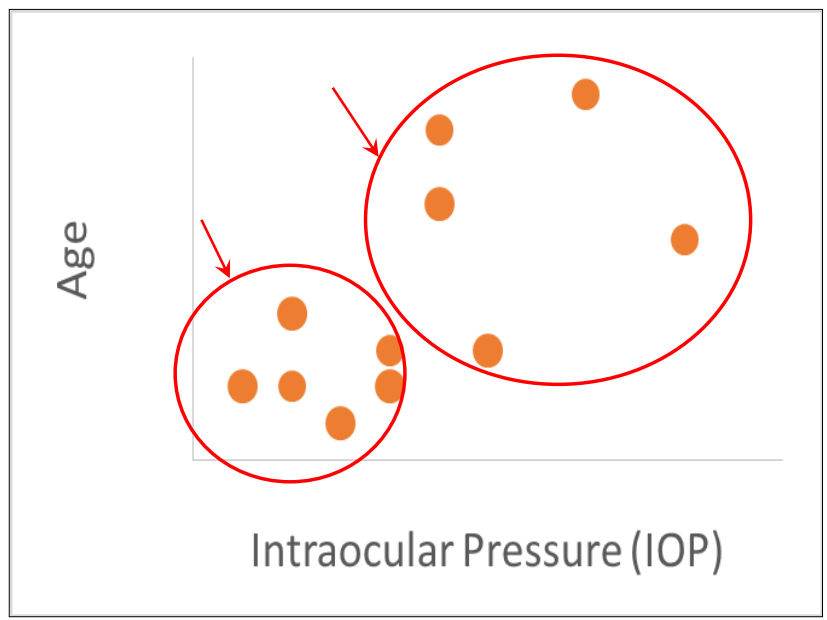

Figure 4: Example of cluster analysis using unsupervised learning.

1.8 Comparison of aql in the Qur'an and Artificial Neural Network

ANN is an example of a learning algorithm with the ability to learn from a set of training examples. This section briefly describes simply the underlying principles in ANN with an attempt to relate the mechanism employed in the algorithm with the Qur'anic concept of aql.

The algorithm is biologically inspired aiming towards finding an optimal model that can mimic a specific output as demonstrated in the previous section of predicting whether the eye is normal or glaucomatous. Figure 5 illustrates a typical ANN structure with two inputs (e.g., IOP and age) and one single output (e.g. 0 for normal or 1 for glaucoma). Note that the connections from bias are removed to avoid clutter. The inputs are connected to nodes and nodes in turn connected to the outputs. Each node represents a mathematical equation for example a sigmoid function which is analogous to the biological dendrite in brain neurons. The node will output a continuous value from 0 to 1 based on the sign and weight of the inputs. The connection thickness represents its weight and the colour determines whether it is a positive or negative value. Thicker connection indicates the information from the input contributes greater contribution and red colour denotes it has a positive influence to the node in the process of making decision. ANN identifies optimal values of the connection weights based on a process called backpropagation. The final node will output the probability of the eye diagnosed as glaucomatous.

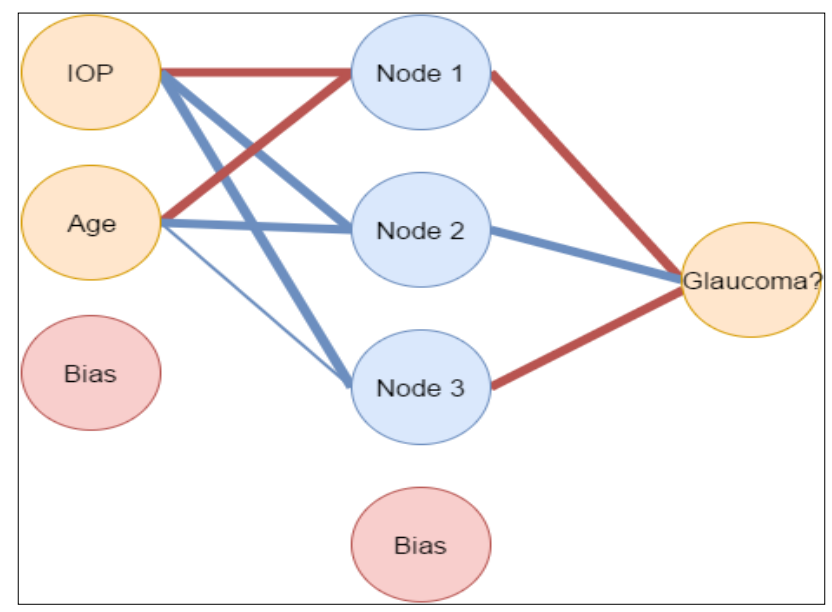

Figure 5: Artificial Neural Network model using hypothetical data of glaucoma diagnosis.

Similar concept of positive and negative similitudes (amsal) can be found in Qur'anic verses with verbs formed from the root word aql which is literally translated as intellect. ${ }^{15}$ While ANN nodes with positive/negative connections are used to map a range of inputs to a specific output, similitudes in the Qur'an suggest that there are instances where aql is not only used in positive but also negative contexts with the final goal of achieving belief (iman) and seeking to be near (yaqin). ${ }^{15}$

\section{RESULTS}

As highlighted in the work of Kocabas (1987), motivation to use the intellect by understanding the signs (ayat) is illustrated in several verses for example:

"Thus does Allah make clear to you His verses that you might use reason." (2:242)

Another example can be found in Surah number 57 verse 17:

"Know that Allah gives life to the earth after its lifelessness. We have made clear to you the signs; perhaps you will understand."

In the same vein, from Surah number 12 verse 2:

"Indeed, We have sent it down as an Arabic Qur'an that you might understand."

Conversely, there are also verses that criticize those who do not activate the intellect. For instance in Surah number 2 verse 144:

"Do you order righteousness of the people and forget yourselves while you recite the Scripture? Then will you not reason?"

Similarly, in Surah number 10 verse 16:

"Say, if Allah had willed, I would not have recited it to you, nor would He have made it known to you, for I had remained among you a lifetime before it. Then will you not reason?"

In the same way, Surah number 39 verse 43 shares the same tone:

"Or have they taken other than Allah

as 
intercessors? Say, even though they do not possess [power over] anything, nor do they reason?"

The evidence found from the verses seems to suggest that similar to ANN, positive and negative instances are used to mould a belief system. However, unlike ANN which has a specific process to assign the strength of the magnitude or importance of each similitude, the process is rather dynamic in shaping a believer - each individual may response differently to the similitude.

\section{The Importance of Human Reason (Aql) in Discovering the Truth of Empirical World: Islamic Perspective}

Islam is religion of knowledge; it urges its followers to seek knowledge by using various tools such as revelation, human reason, observation, experiment, experience and senses in order to discover the truth. It is undeniable fact that Islam from the early beginning perceives human reason $(A q l)$ as a method of learning that is available to seekers of every type of knowledge. Therefore, there are many verses in the holy Qur'an that urge Muslims particularly, and human beings generally to use their 'brain' (the precious gift by Allah to human that distinguishes him from other creations of God) to discover many truths from this visible world.

Basically, Ibn Mandhur argued that, the term al-Aql in Arabic is derived from the verb يعقل . عقل , meaning reason, rationality, intellect or

intelligence, the antonym of which is (stupidity or foolishness). ${ }^{16}$ As such, a naïve person, who acts contrary to his knowledge, deserves not to be called al-Aqil (wise or intelligent). ${ }^{17}$

According to Oxford Dictionary of Current English, reason is the power of being able to think in logical and rational manner. ${ }^{18}$ It means, the ability to think clearly and coherently, or the process of drawing logical inferences.In this sense, reason is an instrument used for examining the coherence and rationality of a body of statements. As such, the examination allows us to conclude that the examined statements are either coherent, and hence in conformity with the principle of reason; or contradictory, and thus in violation of reason. Davutoglu (1994) however, argued that "Reason" represents the human intellectual faculty that processes a variety of information as premises to draw conclusions thereupon. ${ }^{19}$

Whereas, Paul S. Schilling (1948), argues that reason is the thinking activity of man which seeks to discover truth by criticizing, relating, ordering and interpreting, discovering and analyzing coherently the data of consciousness. ${ }^{20}$

It means the premises utilized by the human intellectual faculty and the conclusions drawn thereof can be identified with one or the other discipline of knowledge. Moreover, the human intellectual faculty is not merely considered a processor of information, but is also believed to harbour at least some a priori knowledge consisting of self-evident principles. In other words, it has been argued that a priori knowledge of self-evident principles (also known as the "first principles") is built-in to human intellectual faculty unlike other sources of knowledge (such as revelation, observations, transmitted reports) that are basically external. In short, reason represents the exercise of intellectual faculty in the absence of revealed premises, observations and transmitted reports.

According to Murtadha Mutahari (2014), Qur'an has put a great emphasis on applying reason and wisdom in all matters including gaining the knowledge about physical world, technology, medicine, healthcare sciences, human sciences and social sciences, interpreting history, relationships of various religions, knowing good and evils, justice and injustice, freedom and oppression, nature of this world, moral and immoral and the purpose of this life. ${ }^{21}$ In this regard, the Qur'an is full of references which command and exhort people to think, contemplate and to examine the claims of revelation. It also uses various connotations and words that indicate the importance of utilization of human brain $(A q l)$ in discovering many scientific truths such as, al- ta'qul (thinking), al- tabassur (pondering), al-nazar (seeing or observing), or altadabbur (contemplating) etc.

The following are some Qur'anic verses that endorse the utilization of reason $(A q l)$ for the sake of realization of many truths about physical or metaphysical worlds, as Qur'an emphatically blames those who are reluctant to use this faculty of thinking properly.

1. "Do they not look? At the sky above them? How we made it and adorned it, and there are no flaws in it? And the earth - We have spread it out and set thereon mountains standing firm, and produced therein every kind of Beautiful growth (in pairs). To be observed and commemorated by every devotee turning to God." (50:6-8)

2. "Do they not look at the camels? How they are made? - And at the sky, how it is raised high? - And the mountains, How they are fixed firm? And at the earth, how it is spread out...?" (88:17-20)

3. "Surely the worst of beasts in God's sight are those that are deaf and dumb and do not reason." (8:22)

4. "Have you seen the one who takes as his god his own desire? Then would you be a guardian over him? Or do you think that most of them hear or reason? They are only like cattle; nay, they are even farther astray from the Path." (25:43-44)

5. "And We have certainly created for Hell many of 
the jinn and mankind. They have hearts with which they do not understand, they have eyes with which they do not see, and they have ears with which they do not hear. Those are like livestock; rather, they are more astray. It is they who are the heedless." (7:179)

6. "And it is not for a soul to believe except by permission of Allah, and He will place defilement upon those who will not use reason." (10:100)

In short, from the above verses, it is obvious that, the holy Qur'an does not underestimate the importance of human reason $(A q l)$ in the discovery many truths about our physical world. Yet, it also reminds us that, the intellect is neither the only tool of knowledge; nor the only source of information; because the capacity of the human brain $(A q l)$ is very limited and restricted. In other words, any discovery made by human or the human intellect is relative and imperfect. Indeed, the imperfection of human being and his reason $(A q l)$ is clearly proven by the holy Qur'an when Allah (SWT) says: "O assembly of jinns and men! If you have power to pass beyond the zones of the heavens and the earth, then pass (them)! But you will never be able to pass them, except with authority (from Allah)" (55:33).

\section{CONCLUSION}

We are now at the juncture of understanding a fraction of human learning process and attempting to emulate it. Machine learning based on ANN algorithm is a practical example of inspiration that has been drawn to adopt the learning process derived from the complexity of the human brain and nervous system. The information fed into the ANN helps to regulate its structure by penalising or rewarding each node in the network.

It is shown in this paper, similar concept of positive and negative examples can be observed in Qur'anic verses containing the word aql, where it appears in the context of not only when the intellect is applied but also when not. This suggests that ANN shares similar learning process to achieve belief (iman) by analysing the similitudes (amsal) introduced to the algorithm.

Further deliberation and analysis on other Qur'anic words that share similar connotation with the use of the intellect which include tazakkur, tadabbur, ta'amul, nazar, sam' $u$, and tabassur are needed in the future.

\section{REFERENCES}

1. Kourou K, Exarchos TP, Exarchos KP, Karamouzis MV, Fotiadis DI. Machine learning applications in cancer prognosis and predicion. Comput Struct Biotechnol J 2015; 13:8-17.

2. Cai Z, Xu D, Zhang Q, Zhang J, Ngai SM, Shao J. Classification of lung cancer using ensemblebased feature selection and machine learning methods. Mol Biosyst 2015; 11:791-800.

3. Ng A. Machine learning course. In: Coursera [online]. Available at:

https://www.coursera.org/learn/machinelearning.

4. Campbell M, Hoane AJ, Hsu FH. Deep blue. Artif Intell 2002; 134:57-83.

5. Zastrow M. Google victory at Go stokes Al fear in Korea. New Sci. 2016; 229:9.

6. $\mathrm{M}$. UCI machine learning repository. University of California, Irvine, School of Information and Computer Sciences 2013.

7. Azemin MZC, Kumar DK, Wong TY, et al. Retinal stroke prediction using logistic-based fusion of multiscale fractal analysis. In: 2010 IEEE International Conference on Imaging Systems and Techniques (IST) 2010; 125-128

8. Azemin MZC, Hilmi MR, Mohd Kamal K, et al. Supervised pterygium fibrovascular redness grading using generalized regression neural network. In: New Trends in Software Methodologies, Tools and Techniques. Amsterdam: IOS Press, 2014:650 - 656.

9. Hilmi MR, Che Azemin MZ, Mohd Kamal K, et al. Prediction of Changes in Visual Acuity and Contrast Sensitivity Function by Tissue Redness after Pterygium Surgery. Current Eye Research 2017; 42:852-6.

10. Higuera C, Gardiner KJ, Cios KJ. Selforganizing feature maps identify proteins critical to learning in a mouse model of down syndrome. PLoS One 2015; 10: e0129126.

11. Rögnvaldsson T, You L, Garwicz D. State of the art prediction of HIV-1 protease cleavage sites. Bioinformatics 2014; 31:1204-10.

12. Antal B, Hajdu A. An ensemble-based system for automatic screening of diabetic retinopathy. Knowledge-Based Syst 2014; 60:20-27.

13. Sakar BE, Isenkul ME, Sakar CO, et al. Collection and analysis of a Parkinson speech dataset with multiple types of sound recordings. IEEE J Biomed Heal Informatics 2013; 17:828-834.

14. Isabelle G. Feature Extraction: Foundations and Applications. New York: Springer, 2006.

15. Kocabas S. The Qur'anic concept of intellect. London: The Islamic Philosophical Society, 1987.

16. Manzur I, Mukarram MI. Lisan al-'Arab al-Muhit. Beirut: Dar Lisan al-‘Arab, 1988.

17. Ibn Taymiyyah AA. Risalah fi al-Aql wa al-Ruh. Beirut: Darr al-Hijrah, 1988.

18. Soanes C, Thompson D. The Oxford Dictionary of Current English. Oxford: Oxford University Press, 1992.

19. Davutoglu A. Civilizational Transformation and the Muslim World. Kuala Lumpur: Mahir Publ Sdn Bhd, 1994.

20. Schilling SP. Revelation and Reason. J Bible Relig 1948, 16:13-72.

21. Mutahari A. Understanding the Uniqueness of the Qur'an. Lulu Press, 2014. 